\title{
Helium Droplet-Mediated Deposition and Aggregation of Nanoscale Silver Clusters on Carbon Surfaces
}

Ricardo Fernández-Perea, ${ }^{\dagger}$ Luis F. Gómez, ${ }^{\ddagger}, \#$ Carlos Cabrillo, ${ }^{\dagger}$ Martí Pi, ${ }^{\S}$ Alexander O. Mitrushchenkov, Andrey F. Vilesov, $*,+\infty{ }^{+}$and María Pilar de Lara-Castells*, ${ }^{*}$

${ }^{\dagger}$ Instituto de Estructura de la Materia (C.S.I.C.), Serrano 123, E-28006 Madrid, Spain

${ }^{\ddagger}$ Department of Chemistry, University of Southern California, Los Angeles, California 90089, United States

${ }^{\S}$ Department ECM, Facultat de Física, and IN $^{2}$ UB, Universitat de Barcelona, Diagonal 645, E-08028 Barcelona, Spain

"Université Paris-Est, Laboratoire Modélisation et Simulation Multi Echelle, MSME UMR 8208 CNRS, 5 bd Descartes, 77454

Marne-la-Vallée, France

${ }^{\perp}$ Instituto de Física Fundamental (C.S.I.C.), Serrano 123, E-28006 Madrid, Spain

\section{Supporting Information}

\begin{abstract}
We present experiments and calculations of the deposition and aggregation of silver clusters embedded in helium droplets onto an amorphous carbon surface at room temperature. Calculations were also performed for deposition onto a graphene surface. They involve potentials for the interaction of carbon atoms with silver and helium atoms, provided by $a b$ initio calculations. The numerical simulations were performed for few-nanometer-sized silver clusters including up to $5000 \mathrm{Ag}$ atoms and $\mathrm{He}$ droplets with up to $10^{5}{ }^{4} \mathrm{He}$ atoms. The fluid nature of the ${ }^{4} \mathrm{He}$ droplet is accounted for by the renormalization of the $\mathrm{He}-$ He interaction potential. The numerical results are consistent with deposition experiments with an average number of $3000 \mathrm{Ag}$ atoms per ${ }^{4} \mathrm{He}$ droplet and indicate that the aggregation of the silver clusters on the carbon surface is mediated by secondary droplet impacts. They also reveal nontrivial dynamics of the Ag clusters within the carrier droplet, showing a tendency to drift toward the droplet surface. These findings are of relevance in understanding the heterogeneous deposition patterns (large ramified islands) developed for very large droplets with an average number of Ag atoms per droplet within the million range. Finally, the simulations of large (5000 atoms) Ag cluster deposition on graphene reveals strong superdiffusive behavior. In stark contrast, the diffusion is negligible on the amorphous carbon surface.
\end{abstract}

\section{INTRODUCTION}

Soft landing of free metal clusters onto surfaces at low collision velocity has been discussed in the literature (see, e.g., refs 1 and 2) as an attractive technique for the isolation of nanoclusters for subsequent use in various experiments. Recently, it has been shown that soft deposition can be achieved upon encapsulation in cold $(0.4 \mathrm{~K}) \mathrm{He}$ nanodroplets. ${ }^{3-8}$ It has also been suggested that the $\mathrm{He}$ droplet assembly/deposition technique can be useful in different applications, since metallic and bimetallic core-shell nanoparticles $^{9-14}$ with tunable size, shape, and composition can be produced. For example, a very recent experimental study ${ }^{15}$ has shown that the ${ }^{4} \mathrm{He}$ droplet technique yields a narrow distribution of gold nanoparticles on a $\mathrm{TiO}_{2}$ surface, a system that is known as a catalyst for $\mathrm{CO}$ oxidation and selective oxidation of alcohols. ${ }^{16}$

Recently, ${ }^{17}$ we studied a ${ }^{4} \mathrm{He}$-droplet-mediated deposition process using quantum time-dependent helium density functional-based (TDDFT) calculations as described earlier. ${ }^{18,19}$ We will refer to this work as Paper I. It was found that the collision of gold atoms immersed in a ${ }^{4} \mathrm{He}_{300}$ droplet with a small kinetic energy of less than $0.15 \mathrm{eV}$ per atom with a
$\mathrm{TiO}_{2}(110)$ surface at $T=0 \mathrm{~K}$ can be described as a process fulfilling the conditions characterizing a "soft-landing" process when applied to atoms: sticking probabilities close to unity and the absence of any significant translational motion of the gold atoms during the collision. The ${ }^{4} \mathrm{He}$ droplet carries the gold atom to the minimum of the $\mathrm{Au} /$ surface interaction potential, thus eliminating any translation of $\mathrm{Au}$ atoms on the surface during the impact. In the case of clusters, soft-landing processes are also characterized by minimal structural changes upon deposition. It was shown that the atomic soft-landing process is related to the density fluctuations of the quantum fluid droplet. Remarkably, the classical molecular dynamics (MD) description of the ${ }^{4} \mathrm{He}$ droplet was insufficient to explain the soft deposition of the atomic species. ${ }^{17}$

This article presents some additional measurements and $a b$ initio atomistic simulations addressing the deposition and aggregation of metallic nanoparticles embedded in large ${ }^{4} \mathrm{He}$

\footnotetext{
Received: August 14, 2017

Revised: September 16, 2017

Published: September 19, 2017
} 

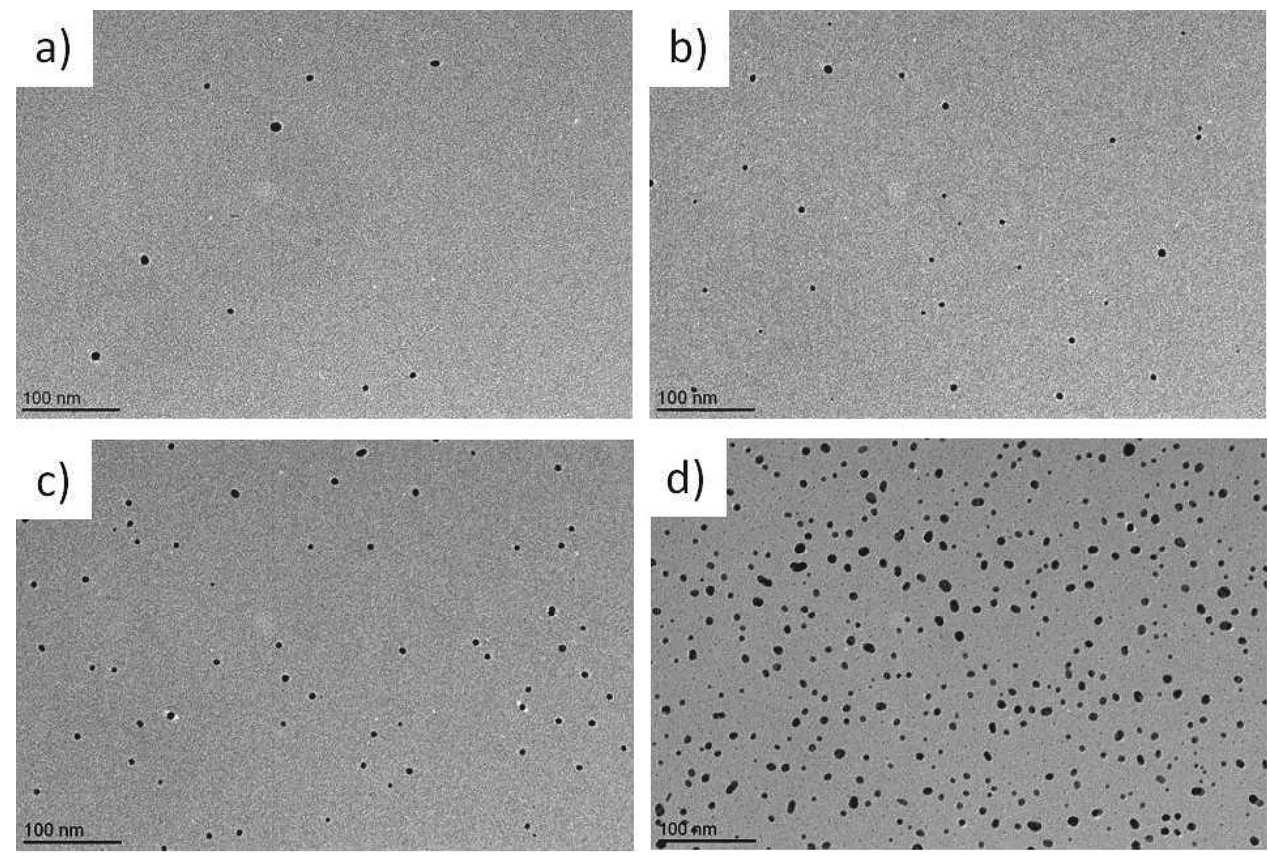

Figure 1. TEM images of $\mathrm{Ag}_{N}$ clusters deposited on a film of amorphous carbon at room temperature. Helium droplets containing $\sim 2.2 \times 10^{7}$ atoms were produced at a nozzle temperature $\left(T_{0}\right)$ of $7 \mathrm{~K}$ and doped with $\sim 3000 \mathrm{Ag}$ atoms. The TEM micrographs were taken at a magnification of $40 \times$ $10^{3}$. The samples were obtained by exposing the surfaces to the doped helium droplet beam during $0.5 \mathrm{~min}(\mathrm{a}), 2 \mathrm{~min}(\mathrm{~b}), 8 \mathrm{~min}$ (c), and $32 \mathrm{~min}$ (d).

droplets. The experiments involve amorphous carbon surfaces, whereas the calculations were performed for graphene and corrugated amorphous carbon. Both experiments and calculations were performed for collisions with a surface at room temperature. The calculations involve Ag clusters consisting of 5000 atoms embedded in ${ }^{4} \mathrm{He}$ droplets of 100000 atoms, whereas larger clusters and droplets were used in the experiments. The calculations address the effect of the interaction with $\mathrm{He}$ atoms of the host $\mathrm{He}$ droplet during the collision as well as the influence of the surface corrugation on the diffusion of the deposited metallic nanoparticles.

The size of the computationally tractable clusters and droplets is limited by the time required for the nuclear dynamics calculations. Due to the large mass, the dynamics of silver nanoparticles can be well described by classical MD simulations once the underlying interatomic interaction forces are known. In contrast, a purely classical description of the motion of the helium atoms is insufficient for a proper description of the quantum fluid ${ }^{4} \mathrm{He}$ droplet. Earlier studies ${ }^{19}$ revealed the spreading of ${ }^{4} \mathrm{He}$ droplets during the impact with a $\mathrm{TiO}_{2}(110)$ surface when quantum TDDFT calculations were performed. Instead, when classical dynamics was applied, a bouncing of the droplet was observed that is inconsistent with the experimentally observed deposition of embedded species. Since the TDDFT treatment become unfeasible for the droplet sizes considered in our simulations (droplets composed of up to $100000{ }^{4} \mathrm{He}$ atoms), we applied the approach proposed by Halberstadt and collaborators ${ }^{20}$ to effectively account for zeropoint effects in classical MD calculations. Very briefly, the scheme introduces a renormalization of the $\mathrm{He}-\mathrm{He}$ interaction potential so that the doped helium droplets are kept liquid, with the bulk value for the density being that of liquid helium (see Section S2 of the Supporting Information for details).

Current calculations employ the interatomic interaction forces that were successfully applied in MD simulations of silver crystals and carbon materials. ${ }^{21,22}$ Interactions of helium nanodroplets and silver clusters with the surface are dominated by van der Waals (vdW) forces. The combination of the incremental method ${ }^{23}$ applied at coupled-cluster level for the long-range dispersion energy component with the dispersionless density functional ${ }^{24}$ for the short-range interaction energy has provided accurate $\mathrm{He} /$ graphene and $\mathrm{Ag}_{2}$ /graphene interaction potentials. ${ }^{25-27}$ These interaction potentials have been used to determine the effective pair $\mathrm{He}-\mathrm{C}$ and $\mathrm{Ag}-\mathrm{C}$ interaction forces, which were then applied in the $\mathrm{MD}$ simulations.

The structure of this article is as follows: Section 2 presents the experimental measurements, whereas Section 3 describes the main computational aspects of the MD simulations. Details of the interaction potentials are provided in the Supporting Information. Section 4 focuses on the discussion of our simulation results and presents a comparison with experiment. Finally, Section 5 contains some concluding remarks and future prospects.

\section{EXPERIMENT}

The $\mathrm{He}$ droplet experiments have been described previously. ${ }^{4,28-31}$ Droplets of average initial sizes $\left\langle N_{\mathrm{He}}\right\rangle=2.2 \times$ $10^{7}$ and $1.7 \times 10^{10}$ were produced by vacuum expansion of the He fluid at a stagnation pressure of $20 \mathrm{bar}$ at $T_{0}=7$ and $5.5 \mathrm{~K}$, respectively. The droplets cool rapidly via the evaporation of ${ }^{4} \mathrm{He}$ atoms and reach a temperature $(0.37 \mathrm{~K})^{32}$ well below the superfluid transition temperature of bulk ${ }^{4} \mathrm{He}$ at $2.17 \mathrm{~K}$. Further downstream, the droplets are doped with $\mathrm{Ag}$ atoms in a hot pick-up cell. The doped ${ }^{4} \mathrm{He}$ droplets collide normally with a substrate that was kept at room temperature located at about 1 $\mathrm{m}$ downstream from the helium droplet source. The doped droplets have a velocity of about $200 \mathrm{~m} / \mathrm{s}$. In the experiments 15-25 nm thick amorphous carbon $(\mathrm{aC})$ substrates were used from Ted Pella Co. $\left(\# 1820^{33}\right)$. These films were manufactured 
by deposition of atomized carbon and have an amorphous structure. After the deposition, the samples were removed from vacuum and imaged by transmission electron microscopy (TEM) within less than $12 \mathrm{~h}$ following the deposition. The imaging was conducted on a JEOL JEM-2100 TEM apparatus at an electron beam energy of $200 \mathrm{keV}$. The TEM images were analyzed with the ImageJ processing package. ${ }^{34}$

As described in ref 4, the average size of the synthesized silver nanoclusters can be estimated from the initial average size of the droplets and the attenuation of the $\mathrm{He}$ droplet beam upon doping. In this work, the average sizes of the silver nanoclusters were $\left\langle N_{\mathrm{Ag}}\right\rangle \sim 3000$ and $2 \times 10^{6}$ in the smaller and larger ${ }^{4} \mathrm{He}$ droplets, respectively. Figure 1 shows TEM images of the samples exposed to the He droplet beam produced at $T_{0}$ $=7 \mathrm{~K}\left(\left\langle N_{\mathrm{Ag}}\right\rangle \sim 3000\right)$ for $0.5,2,8$, and $32 \mathrm{~min}$ in panels $(\mathrm{a})$, (b), (c), and (d), respectively. From Figure 1, it can be seen that the coverage of the samples increases with exposure time. At high exposure, the density of the clusters is higher and the clusters have a larger average size of about $10 \mathrm{~nm}$ across. Some clusters in Figure 1d, obtained at an exposure time of $t=32$ min, have an irregular shape as a result of the coalescence of clusters. Consistent with previous studies, ${ }^{4}$ the TEM images do not show any mobility of the large particles. Smaller clusters, containing a few hundreds of Ag atoms, do display some mobility onto aC surfaces. ${ }^{4}$ Therefore, the observed aggregation is mainly mediated by the accumulation of impacts of successive He droplets via the aggregation of deposited nanoparticles with those embedded in incoming He droplets.

Figure 2 shows the TEM images for samples exposed to the He droplet beam produced at $T_{0}=5.5 \mathrm{~K}\left(\left\langle N_{\mathrm{Ag}}\right\rangle \sim 2 \times 10^{6}\right)$ for $4 \mathrm{~s}, 2 \mathrm{~min}$, and $30 \mathrm{~min}$ in panels (a), (b), and (c), respectively. It can be seen that the deposits obtained under these conditions are much larger in size (up to $100 \mathrm{~nm}$ ) and have some complex morphology. The micrographs also show that the clusters are separated by large areas devoid of noticeable Ag density. As discussed in refs 29 and 35, in large superfluid ${ }^{4} \mathrm{He}$ droplets that contain quantum vortices the deposits consist of elongated metallic wires, which are discernible in Figure $2 \mathrm{~b}$. At a long deposition time of $30 \mathrm{~min}$ (see Figure 2c), the morphology changes remarkably, indicating aggregation of the deposited nanowires and subsequent reconstruction of the deposit in thick structures, with large spaces in between. We notice a large deviation from homogeneity with the emergence of thick filamentous islands separated by large voids. The pattern is reminiscent of some of those observed in systems under complex nonlinear dynamics such as phase separation through spinodal decomposition ${ }^{36-38}$ or Turing patterns in diffusionreaction-like systems. ${ }^{39,40}$ Typically, however, a large mobility is a prerequisite, which is, in turn, affected by competing processes like diffusion-reaction or diffusion-aggregation. Indeed, Ag clusters deposited onto graphitic surfaces, where they show large mobility (see Section 4.3), eventually yield a pattern of fractal $\mathrm{Ag}$ islands owing to a competition between diffusion and aggregation. ${ }^{41}$ In the present scenario of low mobility, however, it is difficult to understand the origin of the pattern. It is most probably due to a combination of many different factors, such as the nature of the substrate, the dynamics of the impact including the excitation of vibrational modes of the carbon substrate, the dynamics of the He droplet over the carbon surface including possible dragging of the Ag clusters, etc.

It could be suggested that the relation between the rate of deposition and the rate of diffusion is another important
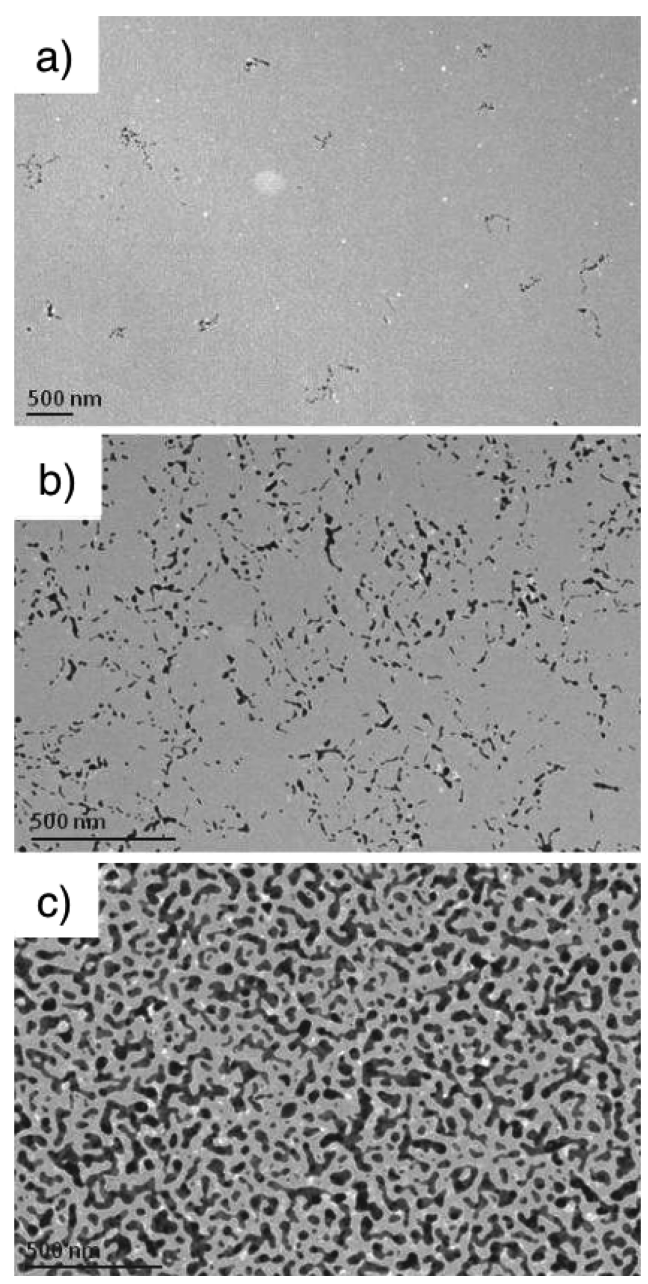

Figure 2. TEM images of $\mathrm{Ag}_{N}$ clusters deposited on a film of amorphous carbon at room temperature. Helium droplets containing $\sim 1.7 \times 10^{10} \mathrm{He}$ atoms were produced at a nozzle temperature $\left(T_{0}\right)$ of $5.5 \mathrm{~K}$ and doped with $\sim 2 \times 10^{6} \mathrm{Ag}$ atoms. Note that the droplets in Figures 2 are 3 orders of magnitude larger than those in Figure 1. The TEM micrographs were taken at magnifications of $10 \times 10^{3}$ for panel (a) and $3 \times 10^{3}$ for panels (b) and (c). The samples were obtained by

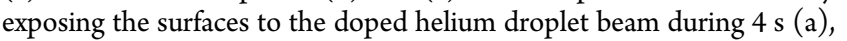
$2 \mathrm{~min}(\mathrm{~b})$, and $30 \mathrm{~min}(\mathrm{c})$.

parameter that may change the morphology of the deposits. Unfortunately, the experimental deposition rate is set by the fluid dynamics of the nozzle expansion to about $5 \times 10^{8}$ and 5 $\times 10^{7}$ droplet collisions per $\mathrm{cm}^{2}$ per second ${ }^{4}$ in Figures 1 and 2, respectively, and cannot be substantially changed in our experiments.

\section{COMPUTATIONAL APPROACH}

To simulate realistic experimental conditions, we performed atomistic simulations relying on accurate interaction potentials and including as many degrees of freedom as possible. As representative nanoparticles, we have chosen clusters composed of 100 and $5000 \mathrm{Ag}$ atoms. The largest cluster size (5000) is characteristic of experiments with readily available smaller $\mathrm{He}$ droplets containing about $10^{7}-10^{8} \mathrm{He}$ atoms ${ }^{4}$ and is close to the average cluster size of $3000 \mathrm{Ag}$ atoms in Figure 1. On the other hand, a study of the impact of very large clusters of about $10^{6} \mathrm{Ag}$ atoms in very large $\mathrm{He}$ droplets of about $10^{10} \mathrm{He}$ atoms remains beyond the reach of the state-of-the-art calculations. Throughout all simulations in this work, the initial temperature 

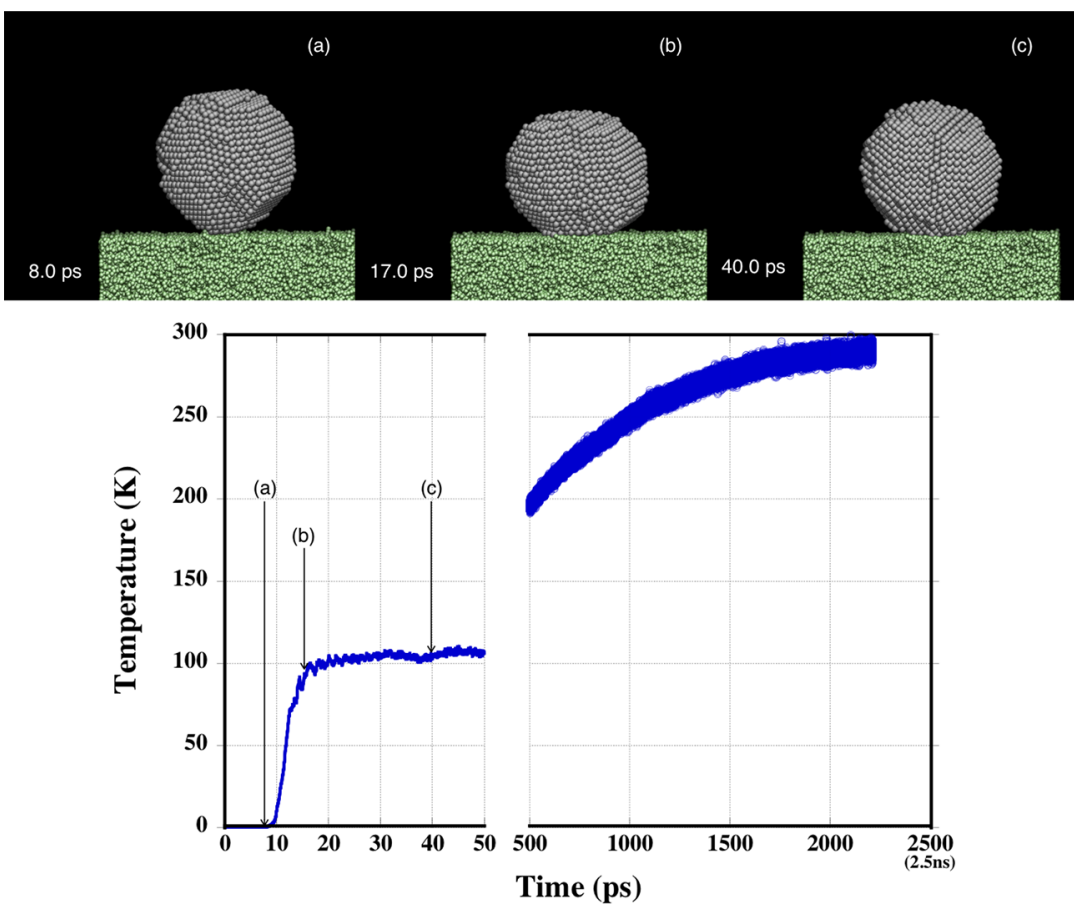

Figure 3. Top panel: Snapshots illustrating the time-dependent evolution of a $\mathrm{Ag}_{5000}$ nanoparticle ( $\mathrm{Ag}$ atoms are shown in gray) at impact with an amorphous carbon surface (C atoms are shown in green), including indications of the associated times: 8 ps (a), 17 ps (b), and 40 ps (c); see also Movie S1. Bottom panel: Evolution of the internal temperature of the $\mathrm{Ag}_{5000}$ nanoparticle.

of the nanoparticles was fixed to $0.4 \mathrm{~K}$ and the impact velocity was set to $200 \mathrm{~m} / \mathrm{s}$ because these represent usual conditions in He droplet-mediated deposition experiments. First, we studied the landing as well as secondary impacts of bare, i.e., not embedded in $\mathrm{He}$ droplets, $\mathrm{Ag}_{5000}$ nanoparticles onto a realistic amorphous carbon structure (see below) at room temperature. Second, the role of the $\mathrm{He}$ droplet in both the landing and secondary impacts processes was addressed by embedding the $\mathrm{Ag}_{5000}$ and $\mathrm{Ag}_{100}$ clusters into $\mathrm{He}$ droplets containing 100000 and $10000{ }^{4} \mathrm{He}$ atoms, respectively. Finally, we analyzed the effect of the surface corrugation; we modeled the deposition and subsequent dynamics of a $\mathrm{Ag}_{5000}$ cluster on a four-layer graphene surface with graphite stacking, i.e., $\mathrm{ABAB}$, in order to simulate a typical experimental graphite surface.

3.1. Molecular Dynamics Simulations. All MD simulations were performed using the LAMMPS (Large-scale Atomic/Molecular Massively Parallel Simulation) package. ${ }^{42}$ Newton's equations of motion were solved through numeric integration using the velocity Verlet algorithm, ${ }^{43}$ with a time step of 1.0 fs. During the whole simulation, the momentum of the center of mass of the carbon atoms was forced to be zero.

With the carbon surfaces in the $x y$ plane, the vertical size (along $z$ coordinate) of the simulation box was $750 \AA$ in all MD calculations. In the case of single bare nanoparticles, the dimension of the simulation box in the $x y$ plane was $100 \AA \times$ $100 \AA$ on the amorphous carbon surface, with about 200000 carbon atoms having been explicitly incorporated in the simulation. The $x y$ area is four times larger in simulations involving the two $\mathrm{Ag}_{5000}$ clusters or ${ }^{4} \mathrm{He}_{10000}$ droplets. In order to properly include the collision dynamics of the ${ }^{4} \mathrm{He}_{100000}$ cluster, the dimension of the simulation box was also increased in the $x y$ plane up to $500 \AA \times 500 \AA$, which also required the inclusion of about 1400000 carbon surface atoms. Atomic coordinates from a realistic amorphous carbon structure of a sample with dimensions $50 \AA \times 50 \AA \times 50 \AA$ were used, as reported by Ricolleau et al. ${ }^{44}$ All simulation boxes were obtained by periodic replicas of this atomic structure in the $x y$ plane. Finally, the simulation of the landing of a $\mathrm{Ag}_{5000}$ nanocluster on a four-layer ( $\mathrm{ABAB}$ stacking) graphene surface was carried out using a simulation box size of $196.76 \AA \times$ $170.71 \AA$ with a C-C bond length of $1.424 \AA$.

The MD simulations were performed in an out-ofequilibrium situation. First, the carbon material was simulated within the Canonical Ensemble using the Berendsen's thermostat $^{45}$ at $T=300 \mathrm{~K}$, which was kept active during the whole simulation. Second, the $\mathrm{Ag}$ clusters and the ${ }^{4} \mathrm{He}$ droplets were independently stabilized until thermal equilibrium was reached at $T=0.4 \mathrm{~K}$, within the Canonical Ensemble, and showed convergence in the density profiles. After the annealing process and stabilization at $0.4 \mathrm{~K}$, we introduced the silver nanoparticles (or the ${ }^{4} \mathrm{He}$ droplet with the silver nanoparticles inside) in the simulation, providing it with a center-of-mass velocity toward the surface of $200 \mathrm{~m} / \mathrm{s}$, as in the experiments (see Section 2). Then, we allowed the nanoparticle (or the doped ${ }^{4} \mathrm{He}$ droplet) to evolve through the associated Newton's equations and to interact with the carbon material. When simulating the landing of a second Ag cluster, the previously deposited Ag cluster along with the carbon atoms of the support had already been thermalized at $T=300 \mathrm{~K}$.

3.2. Interatomic Interaction Forces: Pair Interaction Potentials. The potential obtained via the embedded atom method (EAM) of Foiles et al. ${ }^{21}$ was used to describe the AgAg pair interactions within the Ag clusters. The silver EAM potential was built to fit the experimental data for the bulk metal in the fcc phase such as the sublimation energy, the equilibrium lattice constant, elastic constants, and vacancyformation energies. It well reproduces other properties of silver in solids such as phonon frequencies, surface energies, melting points, and $\mathrm{Ag}$ atomic cohesion energies in finite clusters of various size. The potential also includes contributions for the 
embedding energy of each $\mathrm{Ag}$ atom in the electron density defined by the surrounding $\mathrm{Ag}$ atoms.

In order to describe the interaction between the carbon atoms in the surface, the adaptive intermolecular reactive bond order (AIREBO) potential was employed. ${ }^{22}$ Unlike the classical reactive empirical bond-order (REBO) functional ${ }^{46}$ form, longrange vdW and dihedral terms are included in the AIREBO potential. The AIREBO potential has been shown to well reproduce binding energies and elastic properties of carbon materials. $^{22}$ The LAMMPS implementation of the AIREBO potential employes a $\sigma_{\mathrm{C}-\mathrm{C}}$ value of $3.5 \AA$ for the $\mathrm{vdW}$ term (Lennard-Jones-type) with the usual Verlet cutoff of the potential at a distance of $2.5 \times \sigma_{\mathrm{C}-\mathrm{C}}(8.75 \AA)$.

As shown in the Supporting Information (see Sections S1 and S2), the previously validated $\mathrm{Ag}_{2} /$ graphene ${ }^{25}$ and $\mathrm{He} /$ graphene $^{26,27}$ interaction potentials were used to calculate the silver nanoparticle/surface and helium droplet/surface force fields. The dispersion energies of these vdW-dominated interactions were obtained at the coupled-cluster single, double, and perturbative triples $[\operatorname{CCSD}(\mathrm{T})]$ level. The short-range interaction was described via the dispersionless density functional approach, ${ }^{24}$ which was shown to provide highly accurate potentials when combined with the $\operatorname{CCSD}(\mathrm{T})$ treatment of the dispersion part. ${ }^{25-27}$ The parameters of the of the effective pair $\mathrm{Ag}-\mathrm{C}$ and $\mathrm{He}-\mathrm{C}$ interactions were extracted from these $\mathrm{Ag}_{2} /$ graphene ${ }^{25}$ and $\mathrm{He} /$ graphene ${ }^{26,27}$ interaction potentials. To model the interaction of ${ }^{4} \mathrm{He}$ droplets with silver nanoparticles inside, we fitted the results of very accurate CCSDT calculations of the pair $\mathrm{Ag}-\mathrm{He}$ interaction, ${ }^{47}$ including a variational treatment of triples excitations, to a highly flexible form (see Section S3 in the Supporting Information). Finally, in order to include the ${ }^{4} \mathrm{He}$ droplet dynamics into the MD simulations, we applied a simplified technique following the method proposed by Bonhommeau et al., ${ }^{20}$ as described in detail in the Supporting Information.

\section{RESULTS AND DISCUSSION}

4.1. Landing and Secondary Impacts of Bare Silver Nanoparticles on Amorphous Carbon. The dynamical simulations start with the landing of a pure $\mathrm{Ag}_{5000}$ nanoparticle on the amorphous carbon surface, which models the atomistic structure of realistic TEM support ${ }^{44}$ thermalized at room temperature. After an annealing process and stabilization at 0.4 $\mathrm{K}$, the silver nanoparticle is provided with an impact velocity of $200 \mathrm{~m} / \mathrm{s}$ (as in the He droplet experiments) toward the surface, which is located at $25 \AA$ from the proximal edge of the nanoparticle. This initial distance of $25 \AA$ is large enough for the nanoparticle to be free from any interaction with the carbon surface at the initial time. The same initial distance between proximal edges is considered in all simulations. All carbon atoms were allowed to relax, and no predetermined structure was assumed for the silver nanoparticle. However, an initial (icosahedral) structure was formed through the annealing and stabilization processes.

Figure 3 shows the evolution of the nanoparticle/surface system (see Movie S1). Figure 4 presents the evolution of the center-of-mass velocity of the cluster. The silver nanoparticle sticks to the surface at the low velocity of $200 \mathrm{~m} / \mathrm{s}$, whereas at a higher velocity of $1000 \mathrm{~m} / \mathrm{s}$, the particle melts upon impact, spreading as a thin film. After sticking, the nanoparticle does not diffuse, rebounds a little, and suffers some deformation until thermalizing on the surface. The bottom panel of Figure 3 shows the internal temperature of the nanoparticle as a function

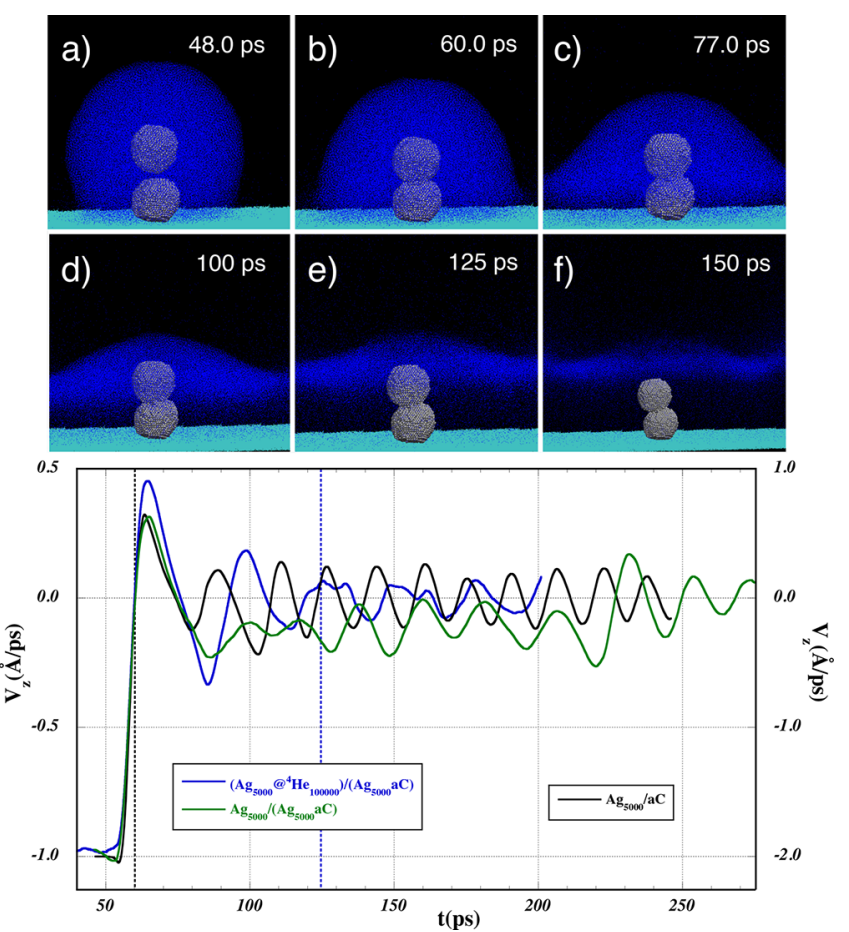

Figure 4. Top panel: Snapshots showing the evolution of an $\mathrm{Ag}_{5000}$ cluster embedded in an ${ }^{4} \mathrm{He}_{100000}$ droplet (Ag atoms are shown in gray; $\mathrm{He}$ atoms are blue) at impact with an $\mathrm{Ag}_{5000}$ cluster previously deposited onto an amorphous carbon surface ( $\mathrm{C}$ atoms are turquoise). Bottom panel: Evolution of the velocity component along the surface normal. The blue line corresponds to the center of mass of the two colliding $\mathrm{Ag}_{5000}$ nanoparticles shown in the top panel. The green line refers to the bare colliding $\mathrm{Ag}_{5000}$ clusters shown in Figure 5, and the black one $\left(\mathrm{V}_{z}\right.$, right $y$ axis $)$ corresponds to the center of mass of the soft landing of the bare $\mathrm{Ag}_{5000}$ nanoparticle displayed in Figure 3. The black dashed line indicates the first passage by zero velocity shifted to 60 ps in all cases, whereas the blue dashed line marks the time associated with snapshot (e), when the He droplet can be considered evaporated. Notice that the scale of the right $y$ axis corresponding to the single nanoparticle is double. With this scaling, the left $y$ axis can also be interpreted as the momentum in $\mathrm{Ag}_{5000}$ mass units for all cases.

of time. It is seen that the sticking occurs in just $10 \mathrm{ps,} \mathrm{similar}$ to that previously found for the deposition of a single gold atom on a titanium dioxide surface. ${ }^{17}$

One fundamental question motivating this work is whether soft-landing conditions are realized in ${ }^{4} \mathrm{He}$ droplet-mediated deposition processes at the nanoscale. Soft landing is usually characterized by the sticking of the nanoparticle to the solid surface at the impact point with a probability near unity while the shape of the particle remains unchanged. Despite some deformation (flattening) of the nanoparticle (see Figure 3), our simulation confirms the existence of a soft landing mechanism. Interestingly, in contrast with the case of a single metallic atom (see Paper $\mathrm{I}^{17}$ ), a bare silver nanoparticle soft lands, which can be explained by its low velocity and temperature. The center-ofmass kinetic energy is dissipated by the plastic deformation of the particle upon impact and other inelastic processes in both the Ag cluster (observe the sharp increment of its temperature in Figure 3) and the amorphous carbon surface. On the other hand, the soft landing of single Ag atoms was observed only for the embedded atoms (see Paper $\mathrm{I}^{17}$ ). In short, the soft landing of nanoclusters is assisted rather than mediated by the helium drop. Our MD simulation results are similar to those previously 
reported for silver ${ }^{48}$ and gold ${ }^{12}$ nanoparticles landing onto the same surface. In previous simulations, however, the interaction with the surface was modeled by a one-dimensional potential, without consideration of its specific atomic structure or atomic dynamics.

As discussed in Section 2, the measurements at different exposure times indicate that the aggregation of silver nanoparticles on amorphous carbon is mediated by the ${ }^{4} \mathrm{He}$ droplets impacting the surface rather than determined by the diffusion of the deposited nanoparticles. In order to obtain further insights into the mechanism of the cluster aggregation on the surface, we modeled the landing of a silver nanoparticle over a similar particle that was previously soft-landed onto the amorphous carbon film. The dynamical simulation starts with the edge of the incoming bare silver nanoparticle at $25 \AA$ from the proximal edge of the previously deposited silver one and with the center of mass also separated by $25 \AA$ (half of the mean nanoparticle radius). Once again, the incoming bare nanoparticle was provided with a velocity of $200 \mathrm{~m} / \mathrm{s}$ pointed toward the surface.

As shown in Figure 5 and Movie S2, immediately after the first contact, a very fast merging process occurs so that a
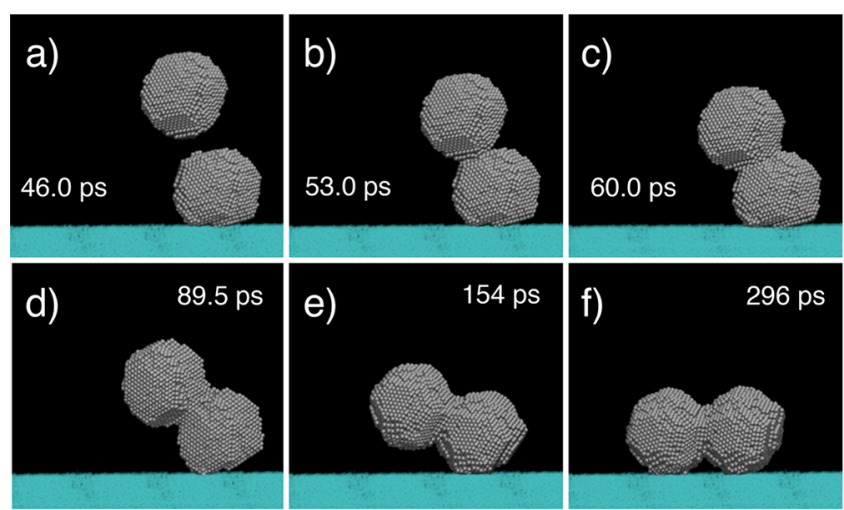

Figure 5. Snapshots showing the evolution of an $\mathrm{Ag}_{5000}$ cluster (Ag atoms are shown in gray) at impact with another $\mathrm{Ag}_{5000}$ cluster previously deposited onto an amorphous carbon $(\mathrm{aC})$ surface with the $\mathrm{C}$ atoms colored turquoise.

merged nanoparticle is formed in just a few picoseconds. This short time scale is similar to that found for the single nanoparticle plastic deformation above. Once merged, the nanoparticle acquires some angular momentum and falls toward the surface in about 220 ps, sticking to it in a horizontal position. This is not necessarily the case, however, if the centerof-mass positions of the incoming and landed nanoparticles are sufficiently well aligned. In such a case, although with a very low probability, we have observed desorption of the composed nanoparticle after the merging process (see Section S4 of the Supporting Information).

4.2. ${ }^{4}$ He-Mediated Deposition of Silver Nanoparticles on Amorphous Carbon. As for the effect of the embedding He droplet, the computational load obviously increases very quickly with the size of the droplet. This is not only due to the large number of $\mathrm{He}$ atoms involved but also to the requirement of increasing the carbon surface area for the purpose of giving the droplet enough space to spread and evaporate. In practical terms, within our computational resources, the largest droplet is composed of $100000 \mathrm{He}$ atoms, requiring simulation box dimensions in the $x y$ plane of $500 \AA \times 500 \AA$. Since this simulation is very time-consuming, we directly studied the secondary impact case. We have altered, however, the initial position of the incoming nanoparticle. Thus, we have aligned the center-of-mass positions of both (incoming and deposited) particles, reproducing exactly the initial conditions under which desorption is observed in the absence of the He drop (see Section S4 of the Supporting Information).

The results are illustrated in the top panel of Figure 4 (see also Movie S3). The bottom panel shows the evolution of the vertical velocity (blue line) compared with both the secondary impact of bare nanoparticles (green line) and the landing of a bare nanoparticle (black line). Once again, as soon as the nanoparticles touch each other, very fast merging occurs. At 60 ps, the already merged nanoparticle starts moving outward from the surface so that the velocity component reaches a value of about $50 \mathrm{~m} / \mathrm{s}$ after 5 ps. The flattening, compression, and spreading of the helium droplet over the surface is evident in the snapshots at 60 and 77 ps. The compression of the helium drop stops the nanoparticle's outward motion so that, at $77 \mathrm{ps}$, it is accelerating toward the surface. In fact, similar to what was previously found for the deposition of a gold atom, ${ }^{17}$ the silver nanoparticle oscillates back and forth with decreasing amplitude. After the ${ }^{4} \mathrm{He}$ droplet evaporates at around $125 \mathrm{ps}$ (vertical blue dashed line in the bottom panel of Figure 4), the oscillations reach a stationary small amplitude around zero. The collision energy is thus effectively dissipated via the evaporation of the $\mathrm{He}$ droplet. As compared with the case of a bare nanoparticle, we first notice that, as expected for the larger angular momentum transfer in the bare impact geometry, the merged particle reaches a larger maximum velocity. Second, falling toward the horizontal position is now much slower so that during the simulation time it cannot be discerned with certainty from a possible vertical libration. In the case of the bare silver particle, the falling is fast enough to dominate the velocity component in the vertical direction so that the oscillations remain negative until the horizontal position is reached at about 225 ps. Notice also that the frequency of the oscillations of the bare single nanoparticle (black line in the bottom panel of Figure 4) scale approximately as $\sqrt{2}$ with respect to the merged counterpart, as expected for thermally driven oscillations in a similar potential well. Finally, when compared with the same impact geometry shown in Section S4 of the Supporting Information, it can be concluded that the ${ }^{4} \mathrm{He}$ droplet effectively cushions the impact, thus inhibiting any possible desorption.

The results of our calculations are consistent with the deposition experiments of $\mathrm{Ag}_{N}$ clusters with $\mathrm{N}_{\mathrm{Ag}}=3000$ (see Figure 1). As mentioned in the Experiment section, the patterns at long exposition times are mainly formed by secondary impacts. It also confirms that the yield is $100 \%$ (i.e., all embedded Ag clusters end up adsorbed on the carbon surface) as estimated in the experimental setups using microbalances. ${ }^{3,4}$ However, the TEM results make clear that some other mechanisms should also contribute. In particular, for the filaments case shown in Figure 2, the patterns are too heterogeneous to arise from just secondary impact accumulation of filaments. Such mechanisms must be related to the effect of the liquid $\mathrm{He}$ droplet on the dynamics of the embedded and/or the already landed particles. In order to better observe this effect, it would be desirable to increase the $N_{\mathrm{He}} / N_{\mathrm{Ag}}=20$ ratio so that it becomes closer to the $10^{4}$ value in the experiments (see Section 2). However, due to the much increased computational load, we instead reduced the overall magnitude of the computation by considering $\mathrm{Ag}_{100}$ silver 
clusters and ${ }^{4} \mathrm{He}_{10000}$ droplets. In this way, the computational load was reduced by more than an order of magnitude while retaining the order of magnitude of the $N_{\mathrm{He}} / N_{\mathrm{Ag}}$ ratio, thus facilitating the exploration of the various scenarios.

We addressed the dynamics of the He droplet-embedded particle when it is not perfectly centered within the droplet, which is a common situation in experiments. For such a task, we prepared an off-centered initial position of the $\mathrm{Ag}_{100}$ cluster, locating it in the plane of the ${ }^{4} \mathrm{He}_{10000}$ sphere parallel to the aC surface but around $25 \AA$ out of the center in the $x$ direction. The diameter of the ${ }^{4} \mathrm{He}_{10000}$ droplet is around $110 \AA$, whereas that of the $\mathrm{Ag}_{100}$ cluster (approximately spherical) is about 18 $\AA$. As usual, the height was such that the proximal edge of the droplet was $25 \AA$ above the $\mathrm{aC}$ surface. As in the experiment, the temperature was fixed to 0.4 and $300 \mathrm{~K}$ for the droplet and the $\mathrm{aC}$ surface, respectively, with the initial droplet velocity being $200 \mathrm{~m} / \mathrm{s}$ downward.

Figure 6 displays the calculated trajectory of the $\mathrm{Ag}_{100}$ centerof-mass position (see also Movie S4). The center of the $\mathrm{He}$

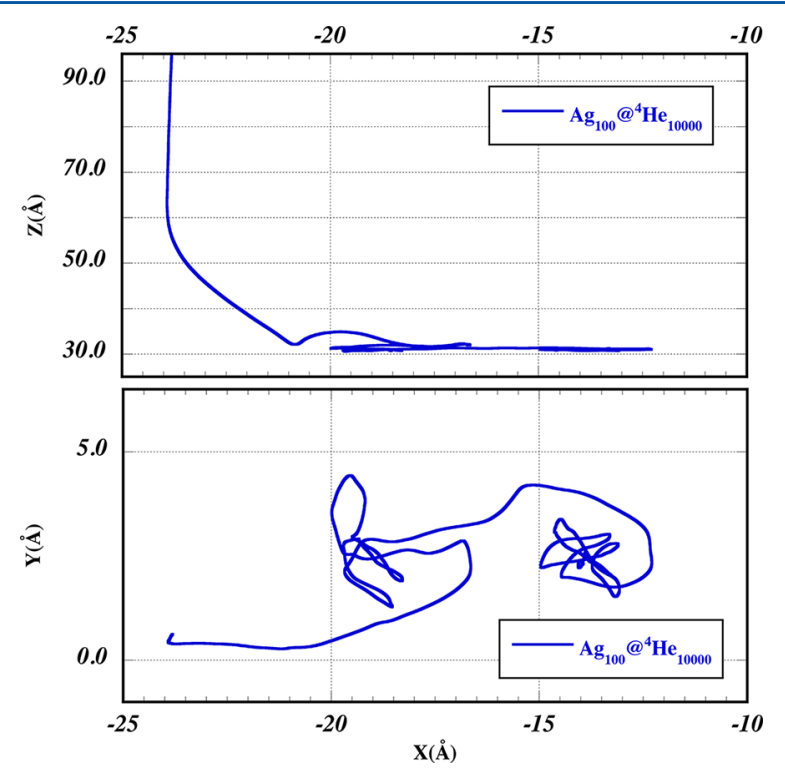

Figure 6. Projections ( $z x$ plane in the top panel; $x y$ plane in the bottom panel) of the center-of-mass trajectory during the landing and posterior diffusion of an off-centered embedded $\mathrm{Ag}_{100}$ cluster in a ${ }^{4} \mathrm{He}_{10000}$ droplet.

droplet is located at about $-50 \AA$ in the $x$ coordinate and close to the origin of the $y$ axis. A drift of the falling trajectory toward the surface of the droplet is very clear. The diffusion of the landed $\mathrm{Ag}_{100}$ cluster is also manifested. The He droplet can be considered evaporated at around the second rebound of the Ag nanoparticle. Notice that the stochastic motion of the landed nanoparticle is rather anisotropic, showing a larger displacement along the $x$ axis. This is consistent with the observed drift because, during the fall, the $\mathrm{Ag}_{100}$ cluster has gained some momentum along the positive $x$ direction. A similar effect would sweep already deposited particles into ramified structures. The study of such a sweeping process will be the subject of future investigations.

These results are illuminating. At this scale, which is orders of magnitude below the experimental $N_{\mathrm{He}} / N_{\mathrm{Ag}}$ ratio, we already observe a clear influence of the $\mathrm{He}$ droplet liquid dynamics onto the embedded Ag nanoparticles, imparting lateral momentum to them that, in turn, facilitates the dragging of already landed $\mathrm{Ag}$ clusters through secondary impacts. Notice that this microscopic mechanism is iteration-dependent: already aggregated clusters are less and less sensitive to a possible pulling/pushing by impinging nanoparticles. As opposed to a pure accumulation of clusters by secondary impacts, here there is also an induced mobility that tends to stabilize the larger aggregates, with the smaller ones remaining eventually mobile. It can be expected that this effect becomes more clearly manifested when operating with a $N_{\mathrm{He}} / N_{\mathrm{Ag}}$ ratio closer to the experimental one. Under these circumstances, a clear heterogeneity in the final $\mathrm{Ag}$ distribution can also be expected. However, at this stage, we are still far from fully understanding the pattern formation, particularly in the filaments case. We have only hints of possible "sources" of heterogeneity. For instance, there is plenty of margin in the $N_{\mathrm{He}} / N_{\mathrm{Ag}}$ ratio to also expect direct dragging by the He droplets in the experimental situations. More speculatively, the helium interaction during the time in which a drop embraces both a falling and a landed nanoparticle could induce some weak effective attraction between the silver nanoparticles. Additionally, the recent observation of the instability of deposited Ag filaments at room temperature due to the self-difussion of $\mathrm{Ag}$ atoms at the surface of the filaments ${ }^{49,50}$ should also have some influence on the shape of the final pattern.

4.3. Diffusion of a Deposited Single Silver Nanoparticle onto Graphene. The influence of the corrugation of surfaces was addressed by substituting the $\mathrm{aC}$ film by a fourlayer graphene sheet ( $\mathrm{ABAB}$ stacking). As described previously (see Section 4.1), after an annealing and stabilization process of the $\mathrm{Ag}_{5000}$ nanoparticle at $0.4 \mathrm{~K}$, it was provided with a velocity toward the surface of $200 \mathrm{~m} / \mathrm{s}$. It can be seen that soft-landing deposition is observed for the $\mathrm{aC}$ surface as well (see Movie S5). However, the diffusive behavior of the deposited Ag cluster is immediately obvious as opposed to the $\mathrm{aC}$ film case where no lateral motion of the deposited particles is observed during the few nanoseconds of the simulations.

The inset of Figure 7 shows the trajectory of the $\mathrm{Ag}_{5000}$ nanoparticle center of mass in the $x y$ plane of the graphene surface during $4.25 \mathrm{~ns}$. At the beginning of a simulation (see Movie S5) associated with rebounding to dampen the impact,

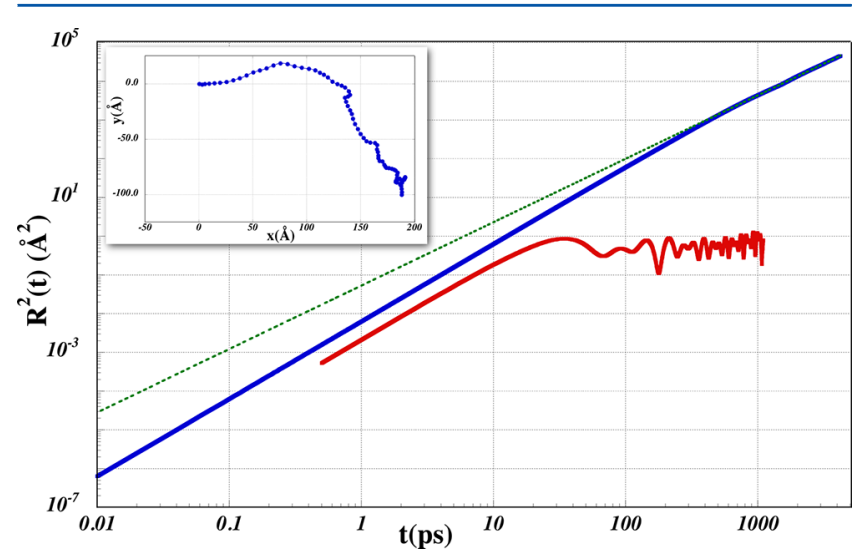

Figure 7. $\log -\log$ plot of the variance of center-of-mass displacement, $R^{2}(t)$, of an $\mathrm{Ag}_{5000}$ cluster as a function of time. Blue thick line: the adsorption surface is a four-layer graphene film at $300 \mathrm{~K}$. The corresponding $4.25 \mathrm{~ns}$ trajectory (displacement in the $x y$ plane) is depicted in the inset. The slope of the function at the long-time limit is shown with a dashed line. Red thick line: the adsorption surface is an amorphous carbon surface at $300 \mathrm{~K}$. 
the nanoparticle rotates along its icosahedral facets. Then, when the nanoparticle settles down, the diffusion in the $x y$ plane starts to dominate the dynamical behavior. In a few nanoseconds, it covers distances of up $200 \AA$.

To better characterize the diffusion process, Figure 7 shows a plot of $\log R^{2}(t)$ vs $\log (\mathrm{t})$, where $R^{2}(t)$ denotes the variance of the displacements as averaged over time origins

$$
R^{2}(t)=\left\langle[\mathbf{R}(t)-\mathbf{R}(0)]^{2}\right\rangle
$$

with $\mathbf{R}$ being the vector defining the center-of-mass position. The $\log R^{2}(t)$ curve at long times $(t \geq 2.5 \mathrm{~ns})$ fits almost perfectly (Pearson's $r=0.999$ ) to a straight line with a slope of 1.63 , i.e., $R^{2}(t) \sim t^{\gamma}$, with $\gamma=1.63$. Remarkably, this is a large departure from the MD results of Luedtke and Landman ${ }^{51,52}$ for the case of gold nanoparticles where a $\gamma$ value of only 1.1 was attained. This represents superdiffusive behavior that is quite strong, closer to the motion of a free particle $(\gamma=2)$ than to the normal diffusion characterized by a linear dependence $(\gamma$ $=1)$. The results are consistent with the observed high diffusion of small $\left(\left\langle N_{\mathrm{Ag}}\right\rangle=150\right)$ clusters onto graphite surfaces. ${ }^{41}$ The superdiffusion behavior is a remarkable effect for such cluster sizes composed of 5000 atoms. As presented in the previous sections, the clusters of the same size show no diffusion onto an $\mathrm{aC}$ surface (al least on the nanosecond scale) so that $\log R^{2}(t)$ remains bounded at long times, i.e., $\gamma=0$ (red line in Figure 7).

\section{CONCLUSIONS}

The main motivation of this combined experimental/ theoretical work was to provide fundamental insights into the mechanisms characterizing ${ }^{4} \mathrm{He}$ droplet-mediated deposition and aggregation of silver nanoparticles on a standard TEM support (amorphous carbon) at room temperature. To this end, we performed MD simulations, including the atoms of the nanoparticle, the droplet, and the support explicitly, applying realistic force fields relying on $a b$ initio determinations of interatomic silver/surface, droplet/surface, and silver/droplet interactions and accounting for leading zero-point effects in the motion of droplets containing up to $100000{ }^{4} \mathrm{He}$ atoms. Moreover, the simulations were extended by also considering a graphene surface.

Deposition experiments with an average number of $\mathrm{Ag}$ atoms per $\mathrm{He}$ droplet of 3000 indicate that the aggregation of the silver clusters on the amorphous carbon surface is mediated by secondary droplet impacts rather than by two-dimensional diffusive motions of the landed nanoparticles. The calculations agree with the experiments in predicting a limited role of twodimensional diffusion. Thus, the simulations clearly show a negligible diffusion of $\mathrm{Ag}$ clusters of 5000 atoms after landing onto the carbon surface, whereas some mobility remains in clusters of 100 atoms. They also characterize the collision process as soft landing even when bare silver clusters are surface deposited at low velocity (about $200 \mathrm{~m} / \mathrm{s}$ ) and temperature (below $0.4 \mathrm{~K}$ ), as in the carrier ${ }^{4} \mathrm{He}$ droplets. With respect to secondary impacts, the simulations reveal a small but finite probability of cluster desorption in the case of bare cluster deposition, a possibility that is inhibited when the clusters are embedded in a He droplet. Remarkably, the simulations also show the possibility of a drift of the embedded cluster toward the surface of the carrier ${ }^{4} \mathrm{He}$ droplet as well as the dragging of deposited particles, as induced by secondary impacts. On the other hand, in counterposition to the amorphous carbon surface, the simulations show not only a mobility of the $\mathrm{Ag}$ clusters of 5000 atoms onto graphitic surfaces but also the onset of a clear superdiffusive regime.

We also show deposition experiments with very large $\mathrm{He}$ droplets and $\mathrm{Ag}$ doping in which the $\mathrm{Ag}$ atoms arrange in large filaments within the He drop. In this case, for a sufficiently long exposure time, the silver aggregates form intriguing patterns of thick filamentous $\mathrm{Ag}$ islands separated by large voids. The scales of such experiments are far larger than the numerical counterparts examined here. Nonetheless, the nontrivial cluster dynamics found in our simulations points toward microscopic mechanisms capable of inducing heterogeneous aggregation. Realistic simulations, such as the ones presented here, will be invaluable in fully understanding such phenomenology. Work in this direction is already underway.

\section{ASSOCIATED CONTENT}

\section{Supporting Information}

The Supporting Information is available free of charge on the ACS Publications website at DOI: 10.1021/acs.jpcc.7b08109.

Details of the pairwise interatomic potential models used in the molecular dynamics simulations, three tables with the model parameters defining the various interatomic pair interactions, and details of the desorption dynamics in central secondary impacts of bare particles (PDF) Movie S1: Simulation of the landing of a nanocluster $\mathrm{Ag}_{5000}$ on an amorphous carbon substrate (AVI)

Movie S2: Simulation of the collision of a nanocluster $\mathrm{Ag}_{5000}$ with a deposited nanocluster $\mathrm{Ag}_{5000}$ onto an amorphous carbon substrate (AVI)

Movie S3: Simulation of the ${ }^{4} \mathrm{He}$ droplet-mediated aggregation of a nanocluster $\mathrm{Ag}_{5000}$ with a deposited nanocluster $\mathrm{Ag}_{5000}$ on an amorphous carbon substrate (the helium droplet is composed of ${ }^{4} \mathrm{He} 100000$ atoms) (AVI)

Movie S4: Simulation of the landing and diffusion of an off-centered nanocluster $\mathrm{Ag}_{100}$ embedded in a ${ }^{4} \mathrm{He}_{10000}$ droplet (AVI)

Movie S5: Simulation of the landing and diffusion of a nanocluster $\mathrm{Ag}_{5000}$ on a graphene sheet (AVI)

Movie S6: Simulation of the central collision of a nanocluster $\mathrm{Ag}_{5000}$ with a deposited nanocluster $\mathrm{Ag}_{5000}$ onto an amorphous carbon substrate (AVI)

\section{AUTHOR INFORMATION}

\section{Corresponding Authors}

*E-mail: vilesov@usc.edu (A.F.V.).

*E-mail: Pilar.deLara.Castells@csic.es (M.P.d.L.-C.).

ORCID

Andrey F. Vilesov: 0000-0002-8412-817X

María Pilar de Lara-Castells: 0000-0001-8697-5770

Present Address

\#(L.F.G.) IPG Photonics, 3939 Freesom Circle, Str. 130, Santa Clara, California 95054, United States.

Notes

The authors declare no competing financial interest.

\section{ACKNOWLEDGMENTS}

This work has been partly supported by the Spanish Agencia Estatal de Investigación (AEI) and the Fondo Europeo de Desarrollo Regional (FEDER, UE) under Grant No. MAT2016-75354-P, the National Science Foundation (United 
States) under Grant Nos. CHE-1362535 and CHE-1664990, and the COST Action CM1405 "Molecules in Motion" (MOLIM). M.P. thanks the finantial support by the AEI and FEDER under Grant No. FIS2014-52285-C2-1-P. M.P.d.L.-C. thanks Andreas W. Hauser, Nadine Halberstadt, and Marius Lewerenz for very helpful discussions. The CTI (CSIC) and CESGA supercomputer facilities (Spain) are acknowledged for the provided resources.

\section{REFERENCES}

(1) Popok, V. N. Energetic Cluster Ion Beams: Modification of Surfaces and Shallow Layers. Mater. Sci. Eng., R 2011, 72, 137-157.

(2) Popok, V. N.; Barke, I.; Campbell, E. E. B.; Meiwes-Broer, K.-H. Cluster-Surface Interaction: From Soft Landing to Implantation. Surf. Sci. Rep. 2011, 66, 347-377.

(3) Mozhayskiy, V.; Slipchenko, M. N.; Adamchuk, V. K.; Vilesov, A. F. Use of Helium Nanodroplets for Assembly, Transport, and Surface Deposition of Large Molecular and Atomic Clusters. J. Chem. Phys. 2007, 127, 094701.

(4) Loginov, E.; Gómez, L. F.; Vilesov, A. F. Surface Deposition and Imaging of Large Ag Clusters Formed in He Droplets. J. Phys. Chem. A 2011, 115, 7199-7204.

(5) Volk, A.; Thaler, P.; Koch, M.; Fisslthaler, E.; Grogger, W.; Ernst, W. E. High Resolution Electron Microscopy of Ag-Clusters in Crystalline and Non-Crystalline Morphologies Grown Inside Superfluid Helium Nanodroplets. J. Chem. Phys. 2013, 138, 214312.

(6) Boatwright, A.; Feng, C.; Spence, D.; Latimer, E.; Binns, C.; Ellis, A. M.; Yang, S. Helium Droplets: a New Route to Nanoparticles. Faraday Discuss. 2013, 162, 113-124.

(7) Emery, S. B.; Rider, K. B.; Little, B. K.; Lindsay, C. M. Helium Droplet Assembled Nanocluster Films: Cluster Formation and Deposition Rates. J. Phys. Chem. C 2013, 117, 2358-2368.

(8) Emery, S. B.; Boyle, J. M.; Rider, K. B.; Little, K. B.; Lindsay, C. M. Nano-scale Energetic Films by Superfluid Helium Droplet Assembly. J. Phys.: Conf. Ser. 2014, 500, 052012.

(9) Thaler, P.; Volk, A.; Lackner, F.; Steurer, J.; Knez, D.; Grogger, W.; Hofer, F.; Ernst, W. E. Formation of Bimetallic Core-Shell Nanowires Along Vortices in Superfluid He Nanodroplets. Phys. Rev. B: Condens. Matter Mater. Phys. 2014, 90, 155442.

(10) Spence, D.; Latimer, E.; Feng, C.; Boatwright, A.; Ellis, A. M.; Yang, S. Vortex-Induced Aggregation in Superfluid Helium Droplets. Phys. Chem. Chem. Phys. 2014, 16, 6903-6906.

(11) Latimer, E.; Spence, D.; Feng, C.; Boatwright, A.; Ellis, A. M.; Yang, S. Preparation of Ultrathin Nanowires Using Superfluid Helium Droplets. Nano Lett. 2014, 14, 2902-2906.

(12) Haberfehlner, G.; Thaler, P.; Knez, D.; Volk, A.; Hofer, F.; Ernst, W. E.; Kothleitner, G. Formation of Bimetallic Clusters in Superfluid Helium Nanodroplets Analyzed by Atomic Resolution Electron Tomography. Nat. Commun. 2015, 6, 8779.

(13) Volk, A.; Thaler, P.; Knez, D.; Hauser, A. W.; Steurer, J.; Grogger, W.; Hofer, F.; Ernst, W. E. The Impact of Doping Rates on the Morphologies of Silver and Gold Nanowires Grown in Helium Nanodroplets. Phys. Chem. Chem. Phys. 2016, 18, 1451-1459.

(14) Buszek, R. J.; Ridge, C. J.; Emery, S. B.; Lindsay, C. M.; Boatz, J. A. Theoretical Study of $\mathrm{Cu} / \mathrm{Mg}$ Core-Shell Nanocluster Formation. J. Phys. Chem. A 2016, 120, 9612-9617.

(15) Wu, Q.; Ridge, C. J.; Zhao, S.; Zakharov, D.; Cen, J.; Tong, X.; Connors, E.; Su, D.; Stach, E. A.; Lindsay, C. M.; et al. Development of a New Generation of Stable, Tunable, and Catalytically Active Nanoparticles Produced by the Helium Nanodroplet Deposition Method. J. Phys. Chem. Lett. 2016, 7, 2910-2914.

(16) Lee, S.; Fan, C.; Wu, T.; Anderson, S. L. CO Oxidation on $\mathrm{Au}_{n} /$ $\mathrm{TiO}_{2}$ Catalysts Produced by Size-Selected Cluster Deposition. J. Am. Chem. Soc. 2004, 126, 5682-5683.

(17) de Lara-Castells, M. P.; Aguirre, N. F.; Stoll, H.; Mitrushchenkov, A. O.; Mateo, D.; Pi, M. Communication: Unraveling the ${ }^{4} \mathrm{He}$ Droplet-Mediated Soft-Landing from $\mathrm{Ab}$ initio-Assisted and
Time-Resolved Density Functional Simulations: $\mathrm{Au} @^{4} \mathrm{He}_{300} /$ $\mathrm{TiO}_{2}(110)$. J. Chem. Phys. 2015, 142, 131101.

(18) Mateo, D.; Hernando, A.; Barranco, M.; Loginov, E.; Drabbels, M.; Pi, M. Translational Dynamics of Photoexcited Atoms in ${ }^{4} \mathrm{He}$ Nanodroplets: the Case of Silver. Phys. Chem. Chem. Phys. 2013, 15, 18388-18400.

(19) de Lara-Castells, M. P.; Stoll, H.; Mitrushchenkov, A. O. Assessing the Performance of Dispersionless and DispersionAccounting Methods: Helium Interaction with Cluster Models of the $\mathrm{TiO}_{2}(110)$ Surface. J. Phys. Chem. A 2014, 118, 6367-6384.

(20) Bonhommeau, D.; Lake, P. T., Jr.; Le Quiniou, C.; Lewerenz, M.; Halberstadt, N. Modeling the Fragmentation Dynamics of Ionic Clusters Inside Helium Nanodroplets: The Case of $\mathrm{He}_{100} \mathrm{Ne}_{4}^{+}$. J. Chem. Phys. 2007, 126, 051104.

(21) Foiles, S. M.; Baskes, M. I.; Daw, M. S. Embedded-AtomMethod Functions for the fcc Metals Cu, Ag, Au, Ni, Pd, Pt, and Their Alloys. Phys. Rev. B: Condens. Matter Mater. Phys. 1986, 33, 79837991.

(22) Stuart, S. J.; Tutein, A. B.; Harrison, J. A. A Reactive Potential for Hydrocarbons with Intermolecular Interactions. J. Chem. Phys. 2000, 112, 6472-6486.

(23) Stoll, H. On the Correlation-Energy of Graphite. J. Chem. Phys. 1992, 97, 8449-8454.

(24) Pernal, K.; Podeszwa, R.; Patkowski, K.; Szalewicz, K. Dispersionless Density Functional Theory. Phys. Rev. Lett. 2009, 103, 263201.

(25) de Lara-Castells, M. P.; Mitrushchenkov, A. O.; Stoll, H. Combining Density Functional and Incremental Post-Hartree-Fock Approaches for van der Waals Dominated Adsorbate-Surface Interactions: $\mathrm{Ag}_{2} /$ Graphene. J. Chem. Phys. 2015, 143, 102804.

(26) de Lara-Castells, M. P.; Stoll, H.; Civalleri, B.; Causà, M.; Voloshina, E.; Mitrushchenkov, A. O.; Pi, M. Communication: A Combined Periodic Density Functional and Incremental WaveFunction-Based Approach for the Dispersion-Accounting TimeResolved Dynamics of ${ }^{4} \mathrm{He}$ Nanodroplets on Surfaces: ${ }^{4} \mathrm{He} /$ Graphene. J. Chem. Phys. 2014, 141, 151102.

(27) de Lara-Castells, M. P.; Bartolomei, M.; Mitrushchenkov, A. O.; Stoll, H. Transferability and Accuracy by Combining Dispersionless Density Functional and Incremental Post-Hartree-Fock Theories: Noble gases Adsorption on Coronene/Graphene/Graphite Surfaces. J. Chem. Phys. 2015, 143, 194701.

(28) Toennies, J. P.; Vilesov, A. F. Superfluid Helium Droplets: A Uniquely Cold Nanomatrix for Molecules and Molecular Complexes. Angew. Chem., Int. Ed. 2004, 43, 2622-2648.

(29) Gómez, L. F.; Loginov, E.; Vilesov, A. F. Traces of Vortices in Superfluid Helium Droplets. Phys. Rev. Lett. 2012, 108, 155302.

(30) Callegari, C.; Ernst, W. E. Handbook of High-Resolution Spectroscopy; John Wiley \& Sons, Ltd, 2011.

(31) Tiggesbaumker, J.; Stienkemeier, F. Formation and Properties of Metal Clusters Isolated in Helium Droplets. Phys. Chem. Chem. Phys. 2007, 9, 4748-4770.

(32) Hartmann, M.; Miller, R. E.; Toennies, J. P.; Vilesov, A. Rotationally Resolved Spectroscopy of $\mathrm{SF}_{6}$ in Liquid Helium Clusters: A Molecular Probe of Cluster Temperature. Phys. Rev. Lett. 1995, 75, $1566-1569$.

(33) Substrates, Support Films for Transmission Electron Microscopy Grids; Ted Pella, Inc.: Redding, CA. http://www.tedpella.com/ Support_Films_html/Support_Films_and_Substrates_Overview. htm\#carbon-type-a.

(34) Schneider, C. A.; Rasband, W. S.; Eliceiri, K. W. NIH Image to ImageJ: 25 years of image analysis. Nat. Methods 2012, 9, 671-675.

(35) Gomez, L. F.; Ferguson, K. R.; Cryan, J. P.; Bacellar, C.; Tanyag, R. M. P.; Jones, C.; Schorb, S.; Anielski, D.; Belkacem, A.; Bernando, C.; et al. Shapes and Vorticities of Superfluid Helium Nanodroplets. Science 2014, 345, 906-909.

(36) Ping, D.; Ohnuma, M.; Hono, K.; Watanabe, M.; Iwasa, T.; Masumoto, T. Microstructures of $\mathrm{FePt}-\mathrm{Al}-\mathrm{O}$ and $\mathrm{FePt}-\mathrm{Ag}$ Nanogranular Thin Films and Their Magnetic Properties. J. Appl. Phys. 2001, 90, 4708-4716. 
(37) Koyama, T.; Onodera, H. Modeling of Microstructure Changes in FePt Nano-Granular Thin Films Using the Phase-Field Method. Mater. Trans. 2003, 44, 1523-1528.

(38) Du, B.; Xie, F.; Wang, Y.; Yang, Z.; Tsui, O. K. Dewetting of Polymer Films with Built-in Topographical Defects. Langmuir 2002, $18,8510-8517$.

(39) Volkening, A.; Sandstede, B. Modelling Stripe Formation in Zebrafish: an Agent-Based Approach. J. R. Soc., Interface 2015, 12, 20150812.

(40) Yizhaq, H.; Bel, G. Effects of Quenched Disorder on Critical Transitions in Pattern-Forming Systems. New J. Phys. 2016, 18, 023004.

(41) Brechignac, C.; Cahuzac, P.; Carlier, F.; Colliex, C.; Leroux, J.; Masson, A.; Yoon, B.; Landman, U. Instability Driven Fragmentation of Nanoscale Fractal Islands. Phys. Rev. Lett. 2002, 88, 196103.

(42) Plimpton, S. Fast Parallel Algorithms for Short-Range Molecular Dynamics. J. Comput. Phys. 1995, 117, 1-19.

(43) Verlet, L. Computer "Experiments" on Classical Fluids. I. Thermodynamical Properties of Lennard-Jones Molecules. Phys. Rev. 1967, 159, 98.

(44) Ricolleau, C.; Le Bouar, Y.; Amara, H.; Landon-Cardinal, O.; Alloyeau, D. Random vs Realistic Amorphous Carbon Models for High Resolution Microscopy and Electron Diffraction. J. Appl. Phys. 2013, 114, 213504.

(45) Berendsen, H. J. C.; Postma, J. P. M.; van Gunsteren, W. F.; DiNola, A.; Haak, J. R. Molecular Dynamics with Coupling to an External Bath. J. Chem. Phys. 1984, 81, 3684-3690.

(46) Brenner, D. W. Empirical Potential for Hydrocarbons for Use in Simulating the Chemical Vapor Deposition of Diamond Films. Phys. Rev. B: Condens. Matter Mater. Phys. 1990, 42, 9458-9471.

(47) Cargnoni, F.; Kuś, T.; Mella, M.; Bartlett, R. J. Ground State Potential Energy Surfaces and Bound States of $M-$ He Dimers $(M=$ $\mathrm{Cu}, \mathrm{Ag}, \mathrm{Au}$ ): A Theoretical Investigation. J. Chem. Phys. 2008, 129, 204307.

(48) Thaler, P.; Volk, A.; Ratschek, M.; Koch, M.; Ernst, W. E. Molecular Dynamics Simulation of the Deposition Process of Cold AgClusters under Different Landing Conditions. J. Chem. Phys. 2014, $140,044326$.

(49) Volk, A.; Knez, D.; Thaler, P.; Hauser, A. W.; Grogger, W.; Hofer, F.; Ernst, W. E. Thermal Instabilities and Rayleigh Breakup of Ultrathin Silver Nanowires Grown in Helium Nanodroplets. Phys. Chem. Chem. Phys. 2015, 17, 24570-24575.

(50) Hauser, A. W.; Schnedlitz, M.; Ernst, W. E. A Coarse-Grained Monte Carlo Approach to Diffusion Processes in Metallic Nanoparticles. Eur. Phys. J. D 2017, 71, 150.

(51) Luedtke, W. D.; Landman, U. Slip Diffusion and Lévy Flights of an Adsorbed Gold Nanocluster. Phys. Rev. Lett. 1999, 82, 3835-3838.

(52) Luedtke, W. D.; Landman, U. Erratum: Slip Diffusion and Lévy Flights of an Adsorbed Gold Nanocluster [Phys. Rev. Lett. 82, 3835 (1999)]. Phys. Rev. Lett. 1999, 83, 1702-1702. 